\title{
Long-range mechanical force in colony branching and tumor invasion
}

\author{
Chin-Lin Guo*a, ${ }^{\text {b }}$, Mingxing Ouyang ${ }^{\mathrm{a}}$, Jiun-Yann $\mathrm{Yu}^{\mathrm{a}}$, Andrew Price ${ }^{\mathrm{c}}$, Jordan Maslov ${ }^{\mathrm{b}}$ \\ ${ }^{\mathrm{a}}$ Department of bioengineering, ${ }^{\mathrm{b}}$ Department of applied Physics, ${ }^{\mathrm{c}}$ Department of electrical \\ Engineering, California Institute of Technology, 1200 E California Blvd., Pasadena, CA USA 91125
}

\begin{abstract}
The most concerned factors for cancer prognosis are tumor invasion and metastasis. The patterns of tumor invasion can be characterized as random infiltration to surrounding extracellular matrix (ECM) or formation of long-range path for collective migration. Recent studies indicate that mechanical force plays an important role in tumor infiltration and collective migration. However, how tumor colonies develop mechanical interactions with each other to initiate various invasion patterns is unclear. Using a micro-patterning technique, we partition cells into clusters to mimic tumor colonies and quantitatively induce colony-ECM interactions. We find that pre-malignant epithelial cells, in response to concentrations of type I collagen in ECM ([COL]), develop various branching patterns resembling those observed in tumor invasion. In contrast with conventional thought, these patterns require long-range $(\sim 600 \mu \mathrm{m})$ transmission of traction force, but not biochemical factors. At low [COL], cell colonies synergistically develop pairwise and directed branching mimicking the formation of long-range path. By contrast, at high [COL] or high colony density, cell colonies develop random branching and scattering patterns independent of each other. Our results suggest that tumor colonies might select different invasive patterns depending on their interactions with each other and with the ECM.
\end{abstract}

Keywords: mechanical force, long-range, extracellular matrix, branching morphogenesis, tumor invasion, spatial pattern

*guochin@caltech.edu; phone 1626 395-5746; cpl.caltech.edu

\section{INTRODUCTION}

There is a general concern that various declared treatments for cancer have not been as successful as expected: the overall mortality rate for cancer has not reduced for the past 40 years. One of the reasons is the lack of control on the most important factors in cancer prognosis: invasion and metastasis. To survive and invade, tumor cells utilize properties inhered from normal cells. For example, several lines of studies indicate a similarity between the stroma invasion of carcinoma cells and the branching morphogenesis of epithelial tubular organs. In both processes, cells branch out from preexisting epithelium to invade the surrounding extracellular matrix (ECM) [1-6]. However, the branching morphogenesis of tubular organs are characterized by regular spatial patterns, whereas the invasion of tumor cells is not [7]. Indeed, studies on branching morphogenesis have shown that this process is tightly regulated by the coordination of environmental and cellular factors such as morphogens, ECM rigidity, proteinases, and genetic changes, to form robust, regular morphology $[1,3,7,8]$. By contrast, tumor cells implement a variety of strategies to invade their primary tissues and metastasize to distant cites. These strategies include hypersensitivity to increment of stroma stiffness [9] and chemotaxis to biochemical factors secreted by stroma cells [10]. In addition, the spreading of tumor cells enables the formation of multiple tumor colonies. These colonies can interact with each other through mechanical and/or biochemical couplings. For example, the motions of cells create traction forces at the ECM [11] that enables long-range mechanical coupling between cells for collective migration, as evidenced by the study of cell motions on deformation substrates [12]. These forces can also alter ECM structures [13] and apply mechanical stress to the environment surrounding tumor cells. The interplay between cells seeking for survival under stress might then activate survival programs to facilitate the evolution and adaptation of malignant cells and the transformation of pre-malignant cells.

Here, we conduct in vitro experiments to study how cell colonies mechanically interact with each other to induce invasion-like behavior. For this purpose, we create a model system where cell-ECM interaction can be quantitatively induced. We seek for a platform where cells can respond to ECM molecules and change their morphology. Through a literature search, we found that type I collagen (COL) has been used to study epithelial tubulogenesis in vivo and in vitro. It was observed that breast epithelial cells differentiate into long-range tubules (length $\geq 400 \mu \mathrm{m}$ ) in COL gels $[14,15]$. Accordingly, adding collagenase or stimulating COL expression can perturb epithelial tubular growth [1618]. In vivo, tubule formation is also known to correlate with COL fibril accumulation [19]. COL forms linear polymers

Biosensing and Nanomedicine IV, edited by Hooman Mohseni, Massoud H. Agahi, Manijeh Razeghi, Proc. of SPIE Vol. 8099, 809903 - (C) 2011 SPIE · CCC code: 0277-786X/11/\$18 · doi: 10.1117/12.911904 
[20]. In principle, linear polymers permit a long-range transmission of force, a suggested mechanism for the creation of tubule-like migratory tracks in collective cancer invasion [13]. These motivate us to use cell-COL interactions as a platform, whereby we study how cell colonies develop mechanical interactions with each other. In this study, we use immortalized Human mammary gland cells (MCF-10A), a cell line that can produce normal epithelial architecture in vitro and has been widely used to study pre-malignant behavior [21], as our model system. We further manipulate two parameters to study mechanical interactions: the distance between colonies $(L)$ and the concentration of COL in ECM ([COL]). To specify the distance between cell colonies, we use micro-patterning technique to create cell traps in agarose gels and cover cells with a layer of ECM gel (Fig. 1). Cells clusters partitioned in the traps are used to mimic colonies. The traps are arranged in a hexagonal configuration to provide a constant distance between adjacent clusters (Fig.1). The initial density and orientations of COL fibers are randomized by ECM self-assembly. Through these procedures, we homogenize the initial mechanical interactions between adjacent clusters.

\section{METHODS AND MATRIALS}

\subsection{Cell Culture and maintenance}

MCF-10A cells were kindly provided by Prof. Anand Asthagiri (Caltech, Chemical Engineering). Cells were maintained in DMEM/Ham's F-12 containing HEPES, L-glutamine, 5\% (v/v) horse serum (Invitrogen-Gibco), $20 \mathrm{ng} / \mathrm{mL}$ epidermal growth factor (EGF, Peprotech), $0.5 \mu \mathrm{g} / \mathrm{ml}$ hydrocortisone (Sigma-Aldrich), $0.1 \mu \mathrm{g} / \mathrm{ml}$ cholera toxin (Sigma-Aldrich), 10 $\mu \mathrm{g} / \mathrm{ml}$ insulin (Sigma-Aldrich), and 1\% penicillin/streptomycin.

\subsection{Preparation of type I collagen (COL) gels}

Collagen gels were prepared using Cultrex ${ }^{\circledR}$ 3-D Culture Matrix ${ }^{\mathrm{TM}}$ Rat Collagen I (R\&D), following the protocol provided by the manufacturer. The stock solution of type I collagen $(5 \mathrm{mg} / \mathrm{ml}$ in $20 \mathrm{mM}$ acetic acid), 10x phosphate buffered saline (PBS), 7.5\% sodium bicarbonate $\left(\mathrm{NaHCO}_{3}\right)$, and water $\left(\mathrm{ddH}_{2} \mathrm{O}\right)$ were mixed to a final concentration of COL $0.1-1.3 \mathrm{mg} / \mathrm{ml}$ in $1 \mathrm{x}$ PBS and neutralized to $\mathrm{pH}=7$ by $7.5 \% \mathrm{NaHCO}_{3}$. According to our measurement, the volume ratio of the required amount of collagen stock solution and $7.5 \% \mathrm{NaHCO}_{3}$ is 5:1.

\subsection{Preparation of perfusion chambers}

To assemble the chambers for live cell imaging, stainless steel plates were fabricated with a hole in their middle; the size and the shape of the hole was designed to match the size and the shape of the coverslip. Nail polish was used to seal the coverslip at the bottom of the plate. To perform perfusion experiments, a PDMS block was used to seal the top of the chamber. Before sealing, two open channels of a diameter of $1 \mathrm{~mm}$ were punched at the PDMS block to allow for the insertion of tubes. One tube was for the perfusion of medium buffered with $5 \% \mathrm{CO}_{2}$ delivered through a humidifier. The other was for the delivery of the wasted medium to a sink. Silicone rubber aquarium sealant was used to seal the PDMS block with the chamber. After the sealant was dry and the chamber was stabilized, the chamber was filled with perfusion medium and gravity was used to maintain the perfusion at a flow rate $\sim 10-100 \mu 1 / \mathrm{min}$.

\subsection{Micro-patterned cell traps}

To specify the initial size and configuration of cell clusters, a protocol modified from the one developed by Nelson et al. [22] was used (Fig. 1). Polydimethylsiloxane (PDMS) stamps were created by soft lithography with a circular shape of a diameter of $200 \mu \mathrm{m}$ and a height of $100 \mu \mathrm{m}$. Each circular trap was separated by a distance varying from $200 \mu \mathrm{m}$ to $1000 \mu \mathrm{m}$. To create the traps, $2 \%$ agarose premixed with $1 \%$ gelatin was boiled and filled in perfusion chambers. Here, gelatin was used to enhance cell trapping (the amount of gelatin used in this study does not induce branching). A PDMS stamp was placed on the top of the boiled agarose and gelatin mixture. The chamber was then left in $4^{\circ} \mathrm{C}$ for $1 \mathrm{~h}$. After the gel formed, the stamps were removed and the traps were filled with MCF-10A cells. Excessive cells were washed away and the chamber was filled with normal or serum-free, growth-free medium. The chamber was then mounted to a microscope stage for timelapse microscopy. After 15-30 min, the medium was replaced by a solution of type I collagen to form a layer of gel on the top of the trapped cell clusters. Once the gel formed, the chamber was sealed and perfused with medium, followed by the resuming of timelapse microscopy.

\subsection{Tmelapse microscopy and image analysis}

Olympus IX71 equipped with automatic XYZ stages (MS-2000, ASI) and piezo-electric objective stages (P-721 Pifoc, Physik Instrumente) were used for multi-position, z-scanning, and auto-focusing timelapse microscopy. An 
environmental chamber was used to maintained humidity, $\mathrm{CO}_{2}$ concentration (5\%), and temperature $\left(37^{\circ} \mathrm{C}\right)$. An Electron-Multiplying CCD camera (ImagEM, C9100-13, Hamamatsu, $512 \times 512$ pixels, water-cooled to $-95{ }^{\circ} \mathrm{C}$ to enhance signal to noise ratio) and Olympus objectives including 40x phase contrast (NA: 0.75), 20x (NA: 0.45), and 10x (NA: 0.3) objectives were used to obtained the images. Metamorph (version 7.7) was used to control the devices and the image acquisition. All the image data were acquired by Metamorph (version 7.7) or Labview programs, and analyzed by Metamorph, Matlab, and ImageJ.

\subsection{Identification of branches at individual clusters}

To identify branches, the periphery of cell cluster was manually traced. A branch was defined if a part of cluster periphery was found continuously out of the trap (Fig. 4A).

\subsection{Computation of the correlation of branching orientations between adjacent clusters}

To compute the correlation of branching between adjacent cell clusters in the traps, a line $M$ was drawn to connect the centers of the two adjacent traps (Fig. 4B). Along the line $M$, branches from each cluster were identified. For each cluster, the branch closest to the line was chosen to compute the correlation. If there was no branch, the correlation was set as zero. Along the chosen branch at one cluster, a line from the center of cluster to the tip of the branch was drawn. The angle $\theta$ between this line and the line $M$ (Fig. 4B) was identified using ImageJ. The angle $\theta^{\prime}$ for the other cluster was then identified using the same procedure. The extent of correlation was defined as $\cos \theta \times \cos \theta$ '. The total score of correlation of one branched cluster was defined as the average of all the correlations it had with its six adjacent clusters.

\section{RESULTS}

\subsection{Cell clusters develop pairwise branching through mechanical coupling at low collagen concentrations}

Through changing $[\mathrm{COL}]$ and $L$, we observed three different patterns. Without the overlay of ECM, cells in the traps formed clusters. We defined this as the no-branching pattern. By exposing cells to COL, they were found to develop branching (see Method for a quantitative definition of branches). At low [COL] ( $0.2 \mathrm{mg} / \mathrm{ml}$ ), cells in individual traps were found to develop branching toward cells in the adjacent traps (Fig. 2, white arrowheads), which we defined as directed branching. Directed branching arose in a pairwise manner. Timelapse microscopy revealed that a single cluster could develop branching toward its neighbors with highly directed cell motions, by which the two clusters connected to each other. Due to the constraint of the traps, adjacent clusters could develop branching toward each other but hardly coalesce with each other within the initial 36h. The branched cluster could then develop branching with another cluster (Fig. 2, time $=09: 46$ and 12:00, the cluster with two white arrowheads). By computing the correlation of branching orientations between adjacent branched clusters (see Method), we defined that clusters exhibit the directed-branching pattern if the average correlation score of branching at branched clusters is larger than 0.5.

\subsection{Cell clusters develop random, uncorrelated branching at high collagen concentrations}

At higher $[\mathrm{COL}](\sim 0.5 \mathrm{mg} / \mathrm{ml})$, we observed the third pattern: the random-branching pattern. In this case, most clusters (>90\%) were found to develop branching but the correlation of branching orientations between adjacent clusters was low $(<0.5)$. Indeed, we often observed that clusters developed branching independent of each other and cells branched out from clusters as scattering (Fig. 3, white arrowheads). The motions of cells were also found to be not directional and un-correlated between adjacent clusters. Over the range of tested configurations $(L=200-1000 \mu \mathrm{m}$ and [COL] $=0-1$ $\mathrm{mg} / \mathrm{ml}$, Fig. 5A), the no-branching pattern occurred if $\lambda>600 \mu \mathrm{m}$, whereas random-branching pattern appeared if $\lambda \leq$ $600 \mu \mathrm{m}$ and $[\mathrm{COL}] \geq 0.5 \mathrm{mg} / \mathrm{ml}$. The absence of branching for $\lambda>600 \mu \mathrm{m}$ suggests that the distance below which cells can mechanically sense each other is $\sim 600 \mu \mathrm{m}$.

\subsection{Force but not morphogen is required for branching formation}

The creation of traction force depends on cell contractility. For epithelial cells, cell contractility is primarily regulated by Rho-associated kinase (ROCK) [15]. To examine if traction force and ROCK activity are required in the induction of branching, cells were perfused with serum-free, growth factor-free medium (to minimize interference from other known biochemical stimulations) containing ROCK inhibitor (Y27632, $10 \mu \mathrm{M}$ ) after the overlay of ECM on the circular traps. After 24h, the frequency of directed branching at individual clusters was measured. We found that inhibiting ROCK did prohibit the formation of directed branching (Fig. 5B). In addition, previous studies suggest that branching in epithelial tubulogenesis is modulated by inhibitory morphogens such as transforming growth factor- $\beta 1$ (TGF $\beta 1$ ) [23]. Here, 
however, treating cells with TGF $\beta 1$ (5 ng/ml, which is known to suppress branching morphogenesis [23]) did not significantly suppress branching as in ROCK inhibition (>90\% of clusters were found to form branching (directed or random) at $[\mathrm{COL}]=0.5 \mathrm{mg} / \mathrm{ml}$ and $L=200-600 \mu \mathrm{m}$ ).

\section{DISSUSION}

\subsection{Synergistic effects in branching and invasion}

We compare our findings with current knowledge of branching pattern formation and tumor invasion. Our first finding is that through COL, branching is induced and directed between clusters, rather than spontaneously arising at individual clusters (corresponding to colonies). This is a non-autonomous behavior. It is different from those observed in chemicalinduced branching, where individual cells form branching in response to morphogens or growth factors such as hepatocyte growth factor and epidermal growth factor [24-27]. Non-autonomous behavior has also been observed in autocrine-regulated branching processes, where individual cells secrete inhibitory morphogens (such as TGF- $\beta$ ) to suppress branching at each other [28]. In this study, however, we find that directed branching is unlikely to be regulated by TGF- $\beta$.

\subsection{Mechanical force in branching initiation and invasion}

Our second finding is that through COL, branching is initiated by a bi-directional, long-range (up to $600 \mu \mathrm{m}$ ) mechanical interaction between clusters. In turn, the bi-directionality forms a spatial anisotropy dictating the sites and the orientation of branches. This mechanism is different from current models where branching is induced and maintained by diffusible, biochemical factors $[8,25]$. Spatial inhomogeneity of mechanical stress has been reported in engineered tubule-like structures, where the pattern of inhomogeneity is correlated with the distribution of branching sites [11]. Such spatial inhomogeneity, however, was found at the periphery of engineered structures and dictated by their initial shape [11]. By contrast, spatial anisotropy of mechanical interaction can emerge spontaneously in initially homogeneous environments, as evidenced by the circular trap experiments.

\subsection{Directed and random branching patterns in branching morphogenesis and tumor invasion}

One critical consideration is whether our results can be applied to branching morphogenesis and tumor invasion in vivo. In this study, directed branching is initiated by mechanical interaction between clusters, whereas in vivo branching is primarily induced by biochemical factors. Nevertheless, in both cases the morphological changes of cells create force. The transmission of force can then evoke mechanical interactions. This makes it likely that the mechanism identified here is applicable to branching morphogenesis in vivo. On the other hand, several lines of evidence suggest that tumor invasion exhibit two distinct patterns. The first is random infiltration of tumor cells to their primary tissues, as observed in most in vitro studies where tumor invasion was initiated by increment of ECM stiffness [7]. The second involves the reorganization of ECM into a tubule-like path which allows collective cancer cell invasion [29, 30]. In particular, the second pattern occurs primarily in COL-rich matrix [13]. These two patterns are consistent with the two branching patterns observed in this study.

\subsection{Conclusion remarks}

In principle, mechanical interaction can be combined with chemical-based patterning processes to enhance the robustness of branching pattern formation. For example, with the autocrine of TGF $\beta$, cells can regulate local branching patterns through the diffusion of morphogens, while using long-range mechanical interactions to align branches at large spatial scales. Furthermore, TGF $\beta$ stimulation is known to enhance COL expression, and the anisotropically assembled, fibrillar form of COL can inhibit cell proliferation through activating discoidin domain receptor [17, 31]. These provide additional couplings among mechanical interaction, biochemical signaling, and cell fate. Aberrant cell proliferation and uncontrolled cell migration might then arise when the coupling between mechanical interaction and biochemical patterning is dysregulated. The most important part of this study is to show how cell colonies select different branching patterns (which resemble the patterns observed in tumor invasion) depending on colony density, inter-colony mechanical interaction, and colony-ECM interaction. Formation of different invasive patterns might lead to distinct selection of therapy. For example, the extent of surgical excision for local infiltration of tumor cells is much easier to estimate than the case where tumor cells have created long-range paths for collective invasion. In summary, we have created a simple platform to quantitatively study how multi-cell colonies develop mechanical interactions with each other. Such platform 
can be used to study or engineer branching morphogenesis in vitro, as well as to study the evolution and adaptation of tumor colonies in the presence of colony interactions.

\section{FIGURES AND CAPTIONS}

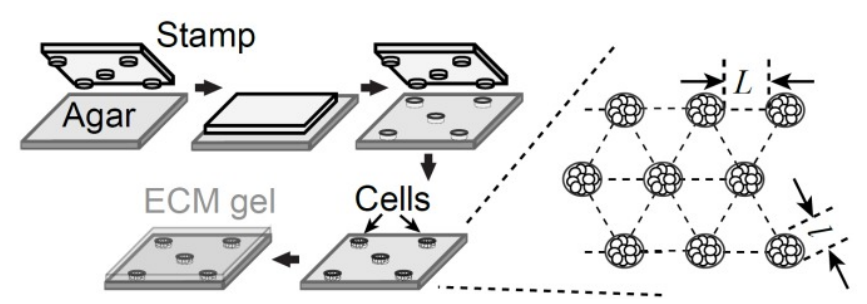

Figure 1. Schematics of circular-trap setup to create cell colonies. Left: A PDMS stamp was used to create circular traps (diameter: $l$; inter-trap distance: $L$; trap depth: $100 \mu \mathrm{m}$ ). Cells were seeded in the traps, followed by the gelification of extracellular matrix (ECM) on top. Right: Schematics of the trap configuration. In this experiment, we used a hexagonal configuration to ensure a constant distance between adjacent traps.
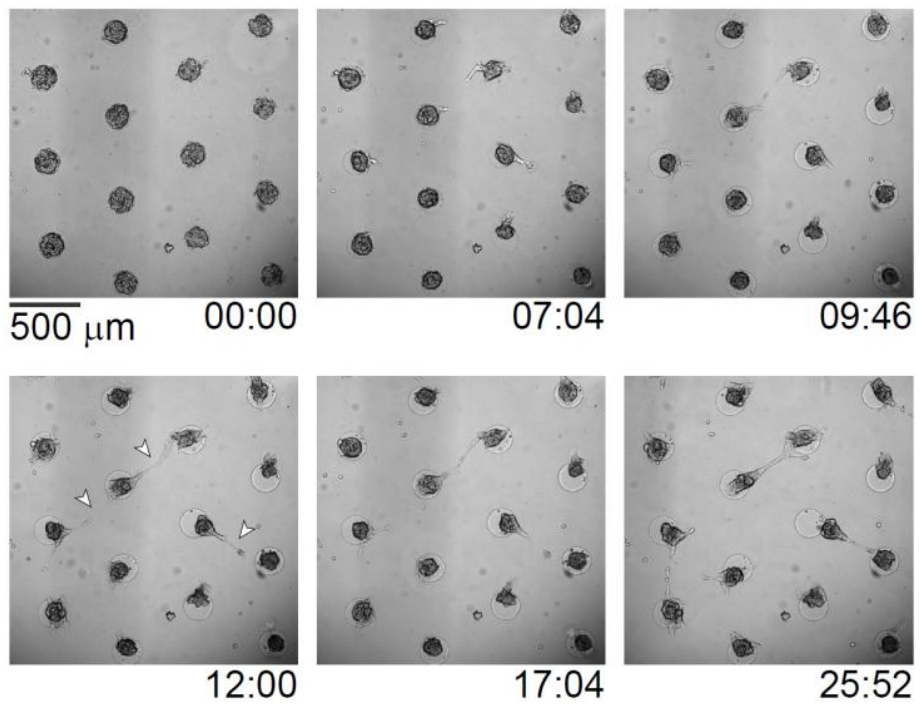

Figure 2. Represented images of directed branching induced by inter-colony interaction. Cells were seeded in the traps (diameter $=200 \mu \mathrm{m}$, inter-trap distance $=400 \mu \mathrm{m}$, and trap depth $=100 \mu \mathrm{m}$ ), followed by the gelification of extracellular matrix (ECM) on top (containing type I collagen $0.2 \mathrm{mg} / \mathrm{ml}$ ). Time is in hour and minute. Time $=00: 00$ is the time for ECM gelification. Note the formation of directed branching between adjacent cell clusters (white arrowhead). 

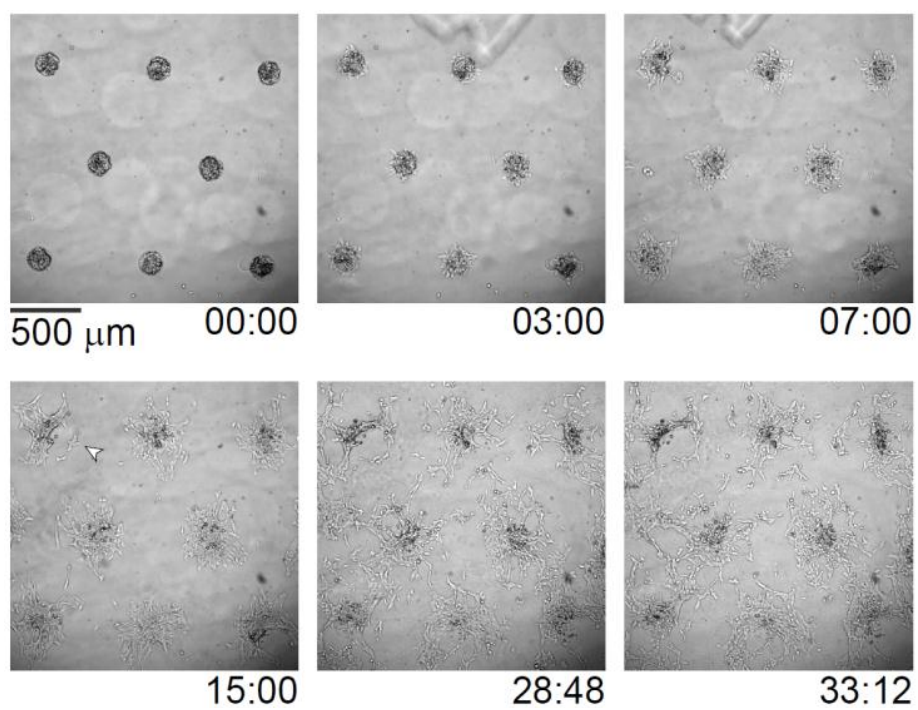

Figure 3. Represented images of random branching induced by high collagen concentrations. Cells were seeded in the traps (diameter $=200 \mu \mathrm{m}$, inter-trap distance $=600 \mu \mathrm{m}$, and trap depth $=100 \mu \mathrm{m}$ ), followed by the gelification of extracellular matrix (ECM) on top (containing type I collagen $0.5 \mathrm{mg} / \mathrm{ml}$ ). Time is in hour and minute. Time $=00: 00$ is the time for ECM gelification. Note the appearance of scattered cells away from the clusters (white arrowhead).
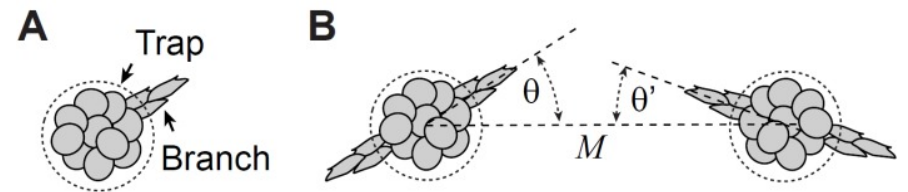

Figure 4. Definitions of branching and branching correlation. A. Cell cluster was defined to have branching if a part of its periphery was out of the trap. B. Angular correlation of branches between adjacent clusters were defined by measuring the angle $\theta$ and $\theta^{\prime}$ along the line $M$. The score of correlation is $(\cos \theta \times \cos \theta$ ') .

$$
\text { A }[\mathrm{COL}]=0.1 \mathrm{mg} / \mathrm{ml}
$$$$
[\mathrm{COL}]=0.3 \mathrm{mg} / \mathrm{ml}
$$
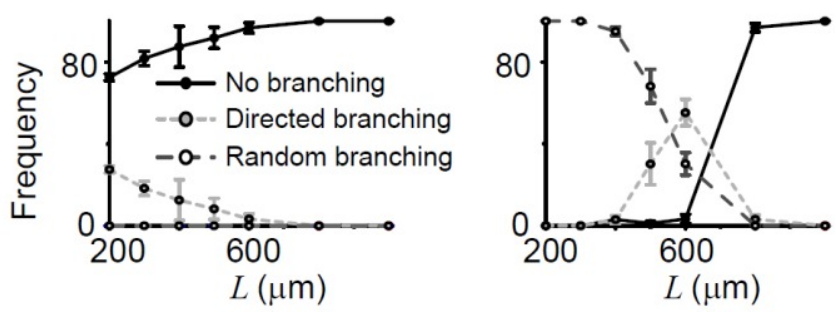

$[\mathrm{COL}]=0.5-1 \mathrm{mg} / \mathrm{ml}$
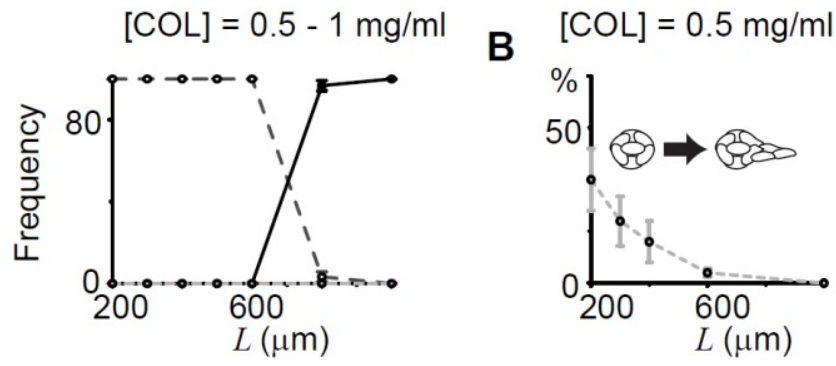

Figure 5. The distribution of branching patterns. A. The distribution of no-branching (black circle), directedbranching (gray circle), and random-branching (white circle) patterns at various colony densities (indicated by $L$ ) and COL concentrations ([COL]). B. The frequency of branching formation (directed or random) at individual clusters in the presence of ROCK inhibitor $(\mathrm{Y} 27632,10 \mu \mathrm{M})$. Data are represented by mean $\pm \mathrm{SEM}$. 


\section{REFERENCES}

[1] J. A. Davies, [Branching Morphogenesis] Landes Bioscience, New York(2006).

[2] A. Horowitz, and M. Simons, "Branching morphogenesis," Circ Res, 103(8), 784-95 (2008).

[3] M. Affolter, R. Zeller, and E. Caussinus, "Tissue remodelling through branching morphogenesis," Nat Rev Mol Cell Biol, 10(12), 831-42 (2009).

[4] J. P. Thiery, H. Acloque, R. Y. Huang et al., "Epithelial-mesenchymal transitions in development and disease," Cell, 139(5), 871-90 (2009).

[5] J. M. Williams, and C. W. Daniel, "Mammary ductal elongation: differentiation of myoepithelium and basal lamina during branching morphogenesis," Dev Biol, 97(2), 274-90 (1983).

[6] A. J. Ewald, A. Brenot, M. Duong et al., "Collective epithelial migration and cell rearrangements drive mammary branching morphogenesis,” Dev Cell, 14(4), 570-81 (2008).

[7] M. J. Paszek, N. Zahir, K. R. Johnson et al., "Tensional homeostasis and the malignant phenotype," Cancer Cell, 8(3), 241-54 (2005).

[8] M. Affolter, S. Bellusci, N. Itoh et al., "Tube or not tube: remodeling epithelial tissues by branching morphogenesis," Dev Cell, 4(1), 11-8 (2003).

[9] S. Munevar, Y. Wang, and M. Dembo, "Traction force microscopy of migrating normal and H-ras transformed 3T3 fibroblasts," Biophysical journal, 80(4), 1744-57 (2001).

[10] O. De Wever, and M. Mareel, "Role of tissue stroma in cancer cell invasion," The Journal of pathology, 200(4), 429-47 (2003).

[11] N. Gjorevski, and C. M. Nelson, "Endogenous patterns of mechanical stress are required for branching morphogenesis," Integr Biol (Camb), 2(9), 424-34 (2010).

[12] T. E. Angelini, E. Hannezo, X. Trepat et al., "Cell migration driven by cooperative substrate deformation patterns," Phys Rev Lett, 104(16), 168104 (2010).

[13] A. Pathak, and S. Kumar, "Biophysical regulation of tumor cell invasion: moving beyond matrix stiffness," Integr Biol (Camb), 3(4), 267-78 (2011).

[14] E. Dhimolea, M. V. Maffini, A. M. Soto et al., "The role of collagen reorganization on mammary epithelial morphogenesis in a 3D culture model," Biomaterials, 31(13), 3622-30 (2010).

[15] M. A. Wozniak, R. Desai, P. A. Solski et al., "ROCK-generated contractility regulates breast epithelial cell differentiation in response to the physical properties of a three-dimensional collagen matrix," J Cell Biol, 163(3), 583-95 (2003).

[16] J. E. Fata, Z. Werb, and M. J. Bissell, "Regulation of mammary gland branching morphogenesis by the extracellular matrix and its remodeling enzymes," Breast Cancer Res, 6(1), 1-11 (2004).

[17] G. B. Silberstein, P. Strickland, S. Coleman et al., "Epithelium-dependent extracellular matrix synthesis in transforming growth factor-beta 1-growth-inhibited mouse mammary gland," J Cell Biol, 110(6), 2209-19 (1990).

[18] M. S. Wicha, L. A. Liotta, B. K. Vonderhaar et al., "Effects of inhibition of basement membrane collagen deposition on rat mammary gland development," Dev Biol, 80(2), 253-6 (1980).

[19] L. Hinck, and G. B. Silberstein, "Key stages in mammary gland development: the mammary end bud as a motile organ," Breast Cancer Res, 7(6), 245-51 (2005).

[20] P. Fratzl, [Collagen: Structure and Mechanics.] Springer, New York(2008).

[21] S. K. Muthuswamy, D. Li, S. Lelievre et al., "ErbB2, but not ErbB1, reinitiates proliferation and induces luminal repopulation in epithelial acini," Nat Cell Biol, 3(9), 785-92 (2001).

[22] C. M. Nelson, M. M. Vanduijn, J. L. Inman et al., "Tissue geometry determines sites of mammary branching morphogenesis in organotypic cultures," Science, 314(5797), 298-300 (2006).

[23] J. V. Soriano, L. Orci, and R. Montesano, "TGF-beta1 induces morphogenesis of branching cords by cloned mammary epithelial cells at subpicomolar concentrations," Biochem Biophys Res Commun, 220(3), 879-85 (1996).

[24] O. B. Garner, K. T. Bush, K. B. Nigam et al., "Stage-dependent regulation of mammary ductal branching by heparan sulfate and HGF-cMet signaling," Dev Biol, (2010).

[25] P. Lu, and Z. Werb, "Patterning mechanisms of branched organs," Science, 322(5907), 1506-9 (2008).

[26] Y. W. Zhang, and G. F. Vande Woude, "HGF/SF-met signaling in the control of branching morphogenesis and invasion," J Cell Biochem, 88(2), 408-17 (2003). 
[27] S. Coleman, G. B. Silberstein, and C. W. Daniel, "Ductal morphogenesis in the mouse mammary gland: evidence supporting a role for epidermal growth factor," Dev Biol, 127(2), 304-15 (1988).

[28] C. W. Daniel, G. B. Silberstein, K. Van Horn et al., "TGF-beta 1-induced inhibition of mouse mammary ductal growth: developmental specificity and characterization,” Dev Biol, 135(1), 20-30 (1989).

[29] K. Wolf, Y. I. Wu, Y. Liu et al., "Multi-step pericellular proteolysis controls the transition from individual to collective cancer cell invasion," Nat Cell Biol, 9(8), 893-904 (2007).

[30] P. Friedl, K. Maaser, C. E. Klein et al., "Migration of highly aggressive MV3 melanoma cells in 3-dimensional collagen lattices results in local matrix reorganization and shedding of alpha 2 and beta 1 integrins and CD44," Cancer Res, 57(10), 2061-70 (1997).

[31] W. F. Vogel, A. Aszodi, F. Alves et al., "Discoidin domain receptor 1 tyrosine kinase has an essential role in mammary gland development," Mol Cell Biol, 21(8), 2906-17 (2001). 